\title{
Noble-Metal-Free Sunlight Harvesting Meta-surface for Water Evaporation
}

\author{
S. Hakuta ${ }^{1,2}$, K. F. MacDonald ${ }^{1}$, and N. I. Zheludev ${ }^{1,3}$ \\ ${ }^{1}$ Optoelectronics Research Centre \& Centre for Photonic Metamaterials, University of Southampton, SO17 1BJ, UK \\ ${ }^{2}$ Frontier Core-Technology Laboratories, Fujifilm, Kanagawa, 258-8577, Japan \\ ${ }^{3}$ Centre for Disruptive Photonic Technologies, Nanyang Technological University, Singapore 637371, Singapore \\ S.Hakuta@soton.ac.uk
}

\begin{abstract}
We present ultrathin multilayer metamaterial absorbers based on abundant, low-cost materials, to effectively harness solar energy for heating and evaporation of water. OCIS codes: (350.6050) Solar energy; (260.2160) Energy transfer; (160.3918) Metamaterials
\end{abstract}

We report on the design and experimental demonstration of ultrathin, broadband meta-surface absorbers for solar energy harvesting and conversion to heat in water for applications such as desalination, distillation, sterilization and purification. Structures are engineered to absorb $>90 \%$ of incident light across a broad wavelength range from 300 to $1540 \mathrm{~nm}$, and to concentrate this energy in the top $100 \mathrm{~nm}$ of the absorber where it can be efficiently transferred to water and thereby harnessed to accelerate evaporation.

There is great and still growing interest in green energy sources that can reduce the collective energy footprint on the environment in the face of accelerating consumption. Solar energy harvesting is well known in the context of photovoltaic technologies but it may also be converted to heat and readily applied to the heating and evaporation of water for desalination, distillation, sterilization and purification, in particular where conventional power sources are unavailable. Such applications require not only an absorber covering as much as possible of the broad UV-NIR solar spectrum but one in which heat is generated and concentrated in a thin surface layer for efficient transfer to surrounding water. 'Perfect' metamaterial absorbers comprising metal/dielectric/nanostructured-metal tri-layers have become a familiar concept in recent years as the basis of resonant and wide-angle absorbers. These are typically based on noble metals, specifically gold and silver, for their strong, low-loss plasmonic properties in the VIS-NIR range but these are expensive metals and not well-suited to high absorption across the full UV-NIR solar spectrum. Here we present broadband meta-surface absorbers based on a low-cost, lossy metal (chromium) as opposed to the noble metals.

a

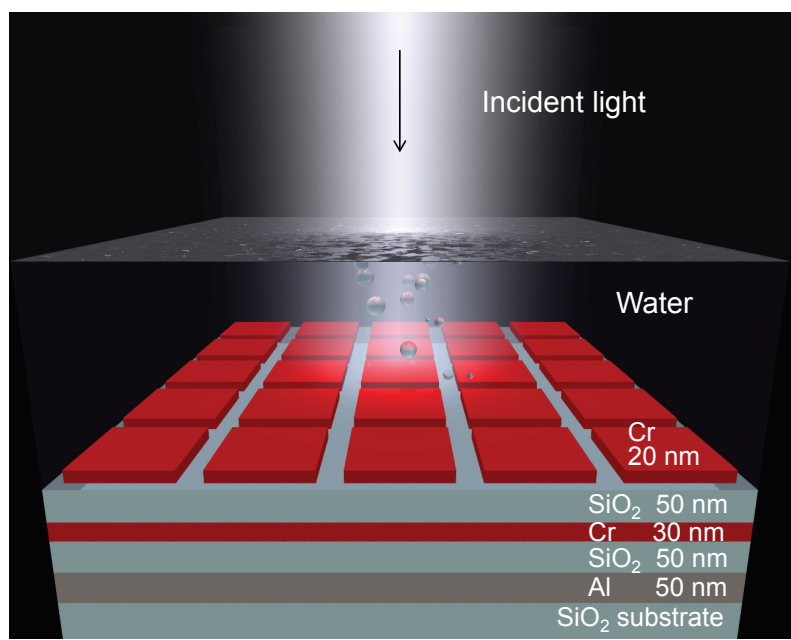

b

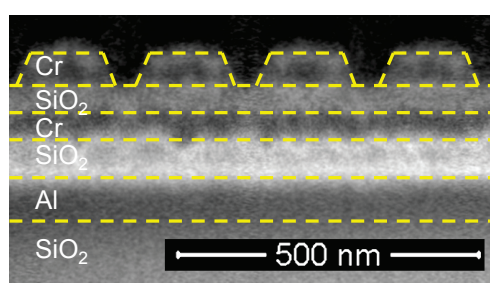

$\mathrm{c}$

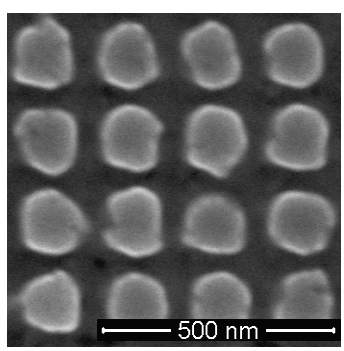

Fig. 1. Sunlight harvesting meta-surface absorber: (a) Schematic illustration of a five-layer $(\mathrm{Al} / \mathrm{SiO} 2 / \mathrm{Cr} / \mathrm{SiO} 2 / \mathrm{structured}-\mathrm{Cr})$ absorber designed for broadband absorption of solar radiation in an aqueous environment and conversion of that energy to heat in the uppermost layers of the stack. (b, c) Scanning electron microscope images (cross-sectional and plan views respectively) of an experimental meta-surface absorber sample with a periodicity of $200 \mathrm{~nm}$ in the top Cr layer.

Through a series of fully three-dimensional finite-element Maxwell solver simulations we evolved a five-layer absorber structure based on chromium $(\mathrm{Cr})$, aluminum $(\mathrm{Al})$ and silica $\left(\mathrm{SiO}_{2}\right)$ as shown in Fig. 1. The electromagnetic analysis assumes monochromatic, plane wave illumination of the structure at normal incidence 
through thin $(400 \mathrm{~nm})$ layer of water; It utilizes established experimental values of the complex dielectric parameters of chromium, aluminum and water [1], while silica is taken to be lossless with a refractive index dispersion as presented in Ref. [2]; By modeling a single translation unit cell of the metamaterial design with periodic boundary conditions in the $x$ and $y$ directions these calculations assume an infinite planar array.

Chromium has a high loss coefficient across a broad wavelength range encompassing the full solar spectrum and sub-wavelength structuring of the top Cr surface layer provides two critical benefits: It modifies the (effective) refractive index of the layer such that reflectivity is reduced and provides for tuning by design of the peak absorption wavelength of the metamaterial (which blue shifts with decreasing period). The nanostructured Cr layer directly absorbs shorter wavelengths while the middle (continuous) Cr layer accounts for longer wavelength components of the solar spectrum. The aluminum provides a small contribution to absorption while acting primarily as a reflector to maximize light absorption, and therefore heat generation, in close proximity to the water interface.

Experimental meta-surface absorbers were prepared on silica substrates by electron beam evaporation, with the top Cr layers subsequently patterned over test areas up to $100 \mu \mathrm{m} \times 100 \mu \mathrm{m}$ by focused ion beam milling. The UVVIS-NIR transmission, reflection and absorption characteristics of samples are quantified in air and in water using a microspectrophotometer (CRAIC QDI2010). Figure 2a shows the sub-aqua absorption spectrum of the five-layer meta-surface structure presented in Fig. 1, alongside the sea-level solar spectrum and, for comparison, the absorption spectrum of a more conventional three-layer metamaterial absorber (structured $\mathrm{Cr}$ and adjacent $\mathrm{SiO}_{2}$ spacer layer exactly as per the 5-layer sample, directly on an Al backplane); The five-layer system absorbs in excess of $90 \%$ of incident light at wavelengths from below 300 to above $1500 \mathrm{~nm}$, confining this energy largely to the top $100 \mathrm{~nm}$ of the multilayer structure as illustrated by the simulated power dissipation density cross-sections in Fig. 2b; The 3layer absorber shows comparable performance at shorter wavelengths up to $900 \mathrm{~nm}$ but is significantly less absorbing in the NIR range.

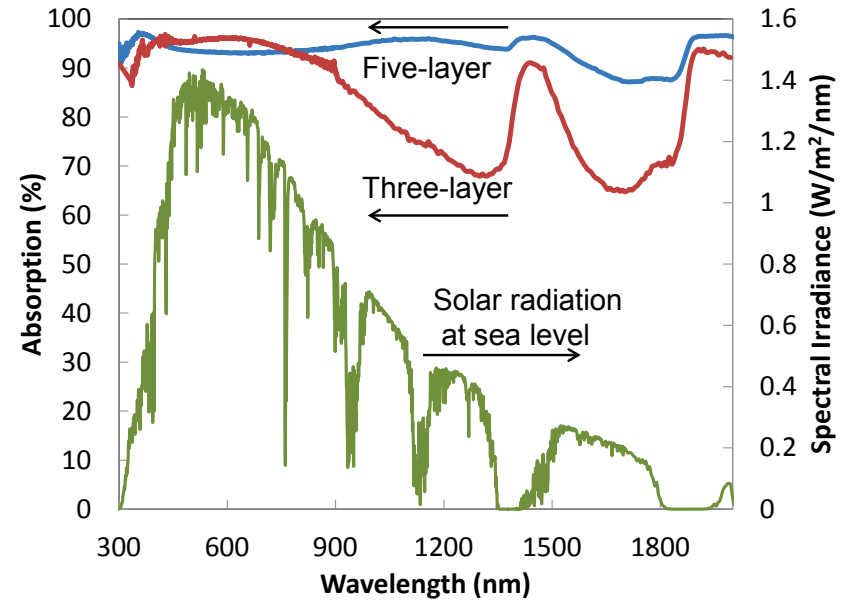

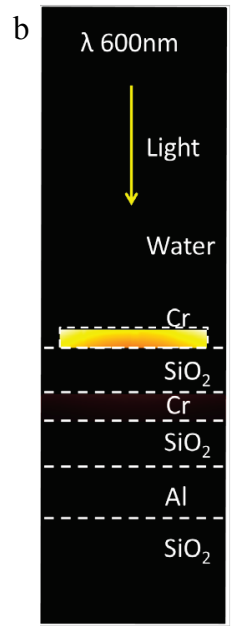

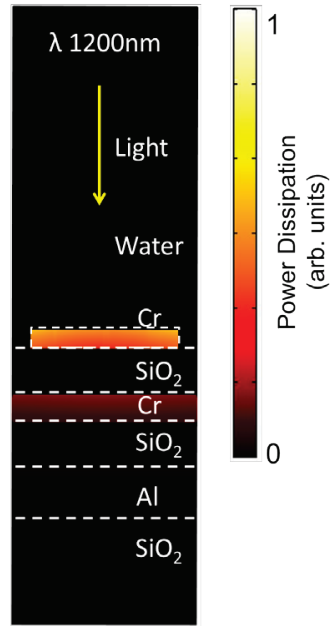

Fig. 2. (a) Solar radiation spectrum at sea level and absorption spectra for five- and three-layer $\mathrm{Cr} / \mathrm{SiO} \mathrm{O}_{2}$ meta-surface absorbers. (b) Numerically simulated power dissipation distributions in the five-layer absorber at illumination wavelengths of 600 and $1200 \mathrm{~nm}$.

In summary, we have engineered meta-surface absorbers, based on abundant, low-cost materials as opposed to the noble metals more commonly employed as the foundation of photonic metamaterial structures, for solar energy harvesting and conversion to heat in water. Such structures may be produced over large area via nano-imprinting methods to serve applications in desalination, distillation, sterilization and water purification.

\section{References}

[1] E. D. Palik, "Handbook of Optical Constants of Solids", Academic Press, (1985); G. M. Hale and M. R. Querry, "Optical Constants of Water in the 200-nm to 200- $\mu \mathrm{m}$ Wavelength Region", Appl. Opt. 12, 555-563 (1973).

[2] I. H. Malitson. "Interspecimen Comparison of the Refractive Index of Fused Silica", J. Opt. Soc. Am. 55, 1205-1209 (1965). 